\title{
Optical properties of silver composite metamaterials
}

\author{
S.M. Orbons ${ }^{\mathrm{a}, *}$, D. Freeman ${ }^{\mathrm{b}}$, B. Luther-Davies ${ }^{\mathrm{b}}$, B.C. Gibson ${ }^{\mathrm{c}}$, S.T. Huntington ${ }^{\mathrm{c}}$, \\ D.N. Jamieson ${ }^{\mathrm{a}}$, A. Roberts ${ }^{\mathrm{a}}$ \\ ${ }^{a}$ School of Physics, University of Melbourne, Victoria 3010, Australia \\ ${ }^{\mathrm{b}}$ Centre for Ultrahigh-bandwidth Devices for Optical Systems, Laser Physics Centre, Australian National University, ACT 0200, Australia \\ ${ }^{c}$ Quantum Communications Victoria, School of Physics, University of Melbourne, Victoria 3010, Australia
}

\begin{abstract}
We present a computational and experimental study investigating the optical properties of nanoscale silver composite metamaterials fabricated by ion beam lithography. Both simulations and experimental results demonstrate high transmission efficiencies in the near infra-red through these devices. Implications for experimentally verifying the calculated near-field distributions of these materials are also discussed.
\end{abstract}

(C) 2007 Elsevier B.V. All rights reserved.

PACS: 78.20.Bh; 81.16.Rf; 42.25.Bs; 42.82.Et

Keywords: Metamaterial; Focused ion beam; Near-field microscopy; Numerical modelling

\section{Introduction}

Recent interest in the optical properties of metamaterials has surged due to their potential applications as negative index material super lenses [1] and biosensors, [2] as well as in the fields of quantum information processing [3] and nonlinear optics [4]. Arrays of annular apertures in thin metallic films show great promise in a number of these fields, due to their high transmission efficiencies at resonant wavelengths [5] and strongly enhanced and localized electromagnetic fields [6]. It has been shown both computationally [5] and experimentally [7] that such metallic composite structures display excellent bandpass characteristics. There are also many reports in the literature of the calculated near-field behaviour of these devices $[6,8,9]$, but there is little experimental evidence published into the investigation of near-field characteristics.

\footnotetext{
*Corresponding author.

E-mail address: sorbons@ph.unimelb.edu.au (S.M. Orbons).
}

\section{Fabrication}

In this study, arrays of annular apertures in thin silver films were fabricated by ion beam lithography. The structures were fabricated by thermally evaporating thin silver films of different thicknesses onto glass substrates with a refractive index of 1.52. An Orsay Physics CANION focused ion beam (FIB) was used to FIB mill arrays of annular apertures in the silver films, using the drift correction technique described in Ref. [10]. Fig. 1 shows micrographs, taken using a Hitachi S4500 field-emission SEM, of a $190 \mathrm{~nm}$ thick sample with a period of $900 \mathrm{~nm}$ and outer and inner diameters of 580 and $330 \mathrm{~nm}$, respectively.

\section{Far-field transmission characteristics}

A rigorous electromagnetic theory developed by Roberts and McPhedran [5] was used to calculate the transmission of electromagnetic fields through a $2 \mathrm{D}$ array of annular aperture in a finite thickness screen of perfectly conducting material. The model describes the fields above the screen and in the infinite substrate below the screen as a 

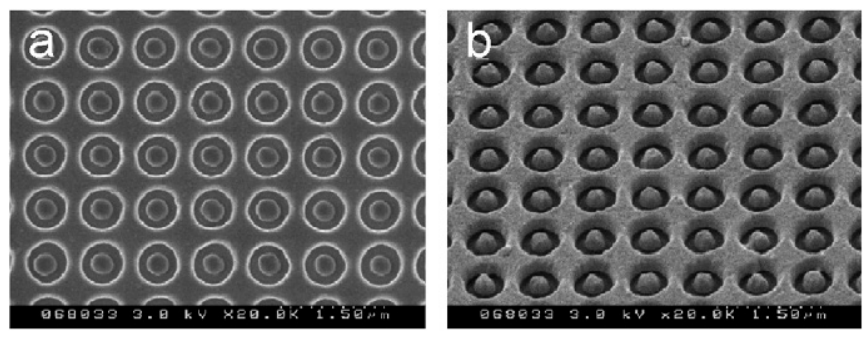

Fig. 1. Images of a $900 \mathrm{~nm}$ period $100 \times 100$ array of annular apertures with an inner and outer diameter of 330 and $580 \mathrm{~nm}$, respectively, in a $190 \mathrm{~nm}$ thick Ag film on a glass substrate: (a) imaged at normal incidence; (b) imaged at $45^{\circ}$ to surface.

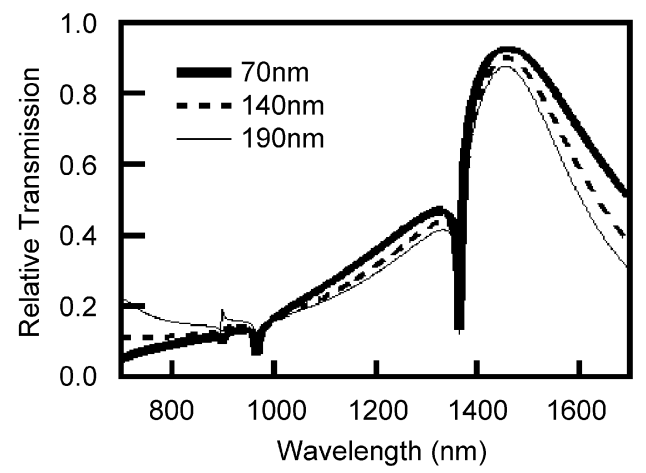

Fig. 2. Calculated transmission spectra for $900 \mathrm{~nm}$ period arrays of annular apertures with outer and inner diameters of 640 and $300 \mathrm{~nm}$, respectively, in perfectly conducting films of thicknesses of 70, 140 and $190 \mathrm{~nm}$.

superposition of plane-waves, whilst expanding the fields within the apertures in coaxial waveguide modes. Fig. 2 illustrates the calculated transmission spectrum for a $900 \mathrm{~nm}$ period array of annular apertures with outer and inner diameters of 640 and $300 \mathrm{~nm}$, respectively, in films with different thicknesses on an infinite substrate with refractive index of 1.52 . The spectra show a zero order transmission peak near $\lambda \approx 1550 \mathrm{~nm}$, as well as a number of diffraction anomalies at wavelengths less than the period multiplied by the substrate index. It is the high transmission efficiency of the zero order peak that makes these devices of interest as band-pass filters. Note that theoretically the location of this peak is approximately equal to the circumference of the rings and its width decreases as the width of the ring becomes narrower [5].

The normal incidence transmission spectra of the devices already described were measured experimentally with the results shown in Fig. 3(b). (Fig. 3(a) shows a schematic illustration of the experimental setup used.) A supercontinuum source was created by passing $1.5 \mathrm{~kW}, 10 \mathrm{ps}$ pulses from a mode-locked $\mathrm{Nd}: \mathrm{YVO}_{4}$ laser $(\lambda=1064 \mathrm{~nm})$ through a $20 \mathrm{~m}$ long section of photonic crystal fibre with zero dispersion at $1040 \mathrm{~nm}$. The spectral range of the resulting beam spanned from below $600 \mathrm{~nm}$ to above $1700 \mathrm{~nm}$. The output from the photonic crystal fibre was subsequently polarized and focused onto the structure with a

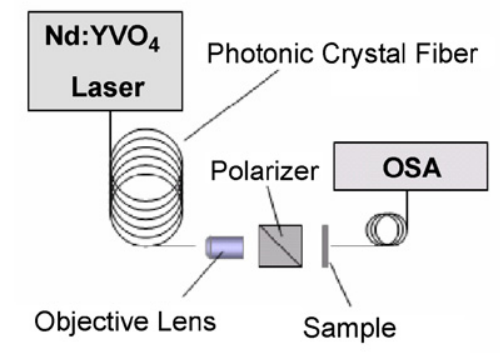

b

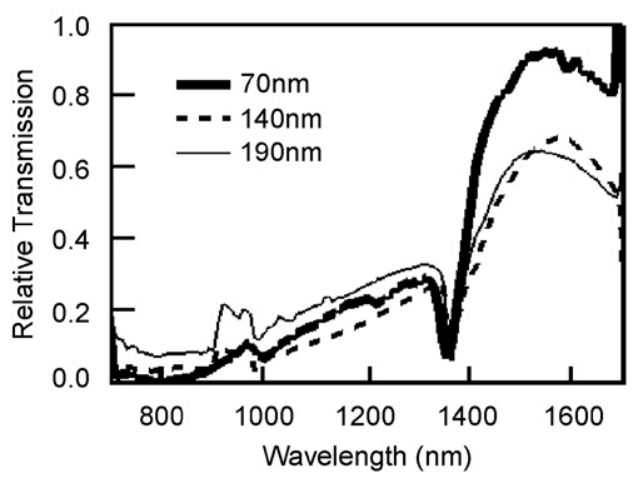

Fig. 3. (a) Schematic illustration of the experimental setup used to measure transmission spectra; (b) measured transmission spectra for $900 \mathrm{~nm}$ period $100 \times 100$ arrays of annular apertures with an inner and outer diameter of 580 and $330 \mathrm{~nm}$, respectively, in silver films of different thickness.

a spotsize of $100 \mu \mathrm{m}$ by a $25 \times$ microscope objective lens. The transmitted fields were then collected by a SMF-28 fibre with $8^{\circ}$ angle polished connector placed closely behind the aperture array and measured with an ANDO AQ6317B optical spectrum analyser (OSA).

Similarities between the experimental and calculated transmission spectra shown in Figs. 2 and $3(\mathrm{~b})$ are immediately obvious. Both reveal diffraction anomalies below $\lambda \approx 1380 \mathrm{~nm}$. More significantly, both display high intensity zero order transmission peaks centred on $\lambda \approx 1550 \mathrm{~nm}$. Interestingly, in the case of the 140 and $190 \mathrm{~nm}$ films, the maximum intensity of the zero order peaks shown in Fig. 3(b) are much lower than the values predicted by the numerical model. However, the measured peak intensity for the $70 \mathrm{~nm}$ sample matches the numerical predictions quite closely. The reason for this inconsistency is most likely due to intrinsic losses caused by material absorption, which is not incorporated into the numerical model. One would expect the effect of material losses to play a greater role as the thickness of the Ag film increases, due to the higher interaction length between the electromagnetic fields and the material. This is indeed what is observed in Fig. 3(b).

Overall, the results shown in Figs. 2 and 3(b) suggest that the modal methods approach outlined earlier describes the essential features of the silver composite structures under investigation in a semi-quantitative fashion. Current work is aimed at the incorporation of intrinsic material losses 
and experimental imperfections into finite difference time domain (FDTD) simulations in order to confirm this result.

\section{Near-field behaviour}

As mentioned earlier, there have been many computational reports in the literature predicting the optical nearfield distribution in films containing annular apertures, but little experimental research addressing this issue has been undertaken. Scanning near-field optical microscopy (SNOM) is a promising tool for providing such evidence, but there are a number of challenges that must be overcome before this can be achieved. Pouet et al. [11] recently demonstrated the use of uncoated probes in characterizing the near-field zones in reflection mode. Here, metal-coated SNOM probes containing a $50 \mathrm{~nm}$ diameter aperture are used to examine the arrays in transmission.

It has been shown $[12,13]$, that metal-coated probes are most sensitive to the component of the magnetic field parallel to the sample surface and the component of the electric field perpendicular to the sample surface. This, along with the fact that there is a strong coupling between the probe and the field means that the interpretation of images obtained using SNOM is very difficult.

FDTD simulations incorporating imperfectly conducting materials are employed to calculate the near-field distribution for structures similar to those outlined earlier. Calculations were performed for composite materials consisting of annular aperture arrays in a $105 \mathrm{~nm}$ thick Ag film, with a period of $1 \mu \mathrm{m}$ and an outer and inner diameter of 500 and $300 \mathrm{~nm}$, respectively, illuminated with a vertically polarized planewave $(\lambda=532 \mathrm{~nm})$. Results from these calculations are shown in Fig. 5.The near-field distribution for a $30 \times 30$ annular aperture array in a $105 \mathrm{~nm}$ thick silver film on a glass substrate $(n=1.52)$ with a $1 \mu \mathrm{m}$ period and an outer and inner diameter of 500 and $300 \mathrm{~nm}$, respectively, was measured using a Nanonics Multiview 1000 near-field scanning optical microscopy system. Fig. 4 shows a schematic illustration of the experimental setup used. The sample was illuminated from the glass substrate side with uncollimated, unpolarized laser radiation $(\lambda=532 \mathrm{~nm})$. The top surface was scanned with a $50 \mathrm{~nm}$ aperture tapered optical fibre (single mode at

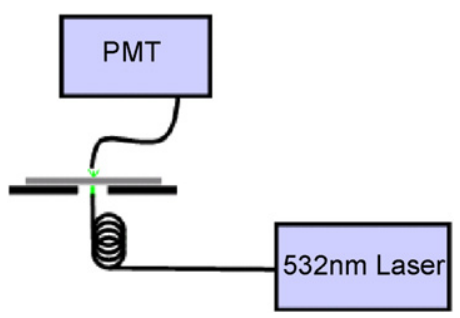

Fig. 4. Schematic illustration of the experimental setup used to measure the near-field distribution using scanning near-field optical microscopy.
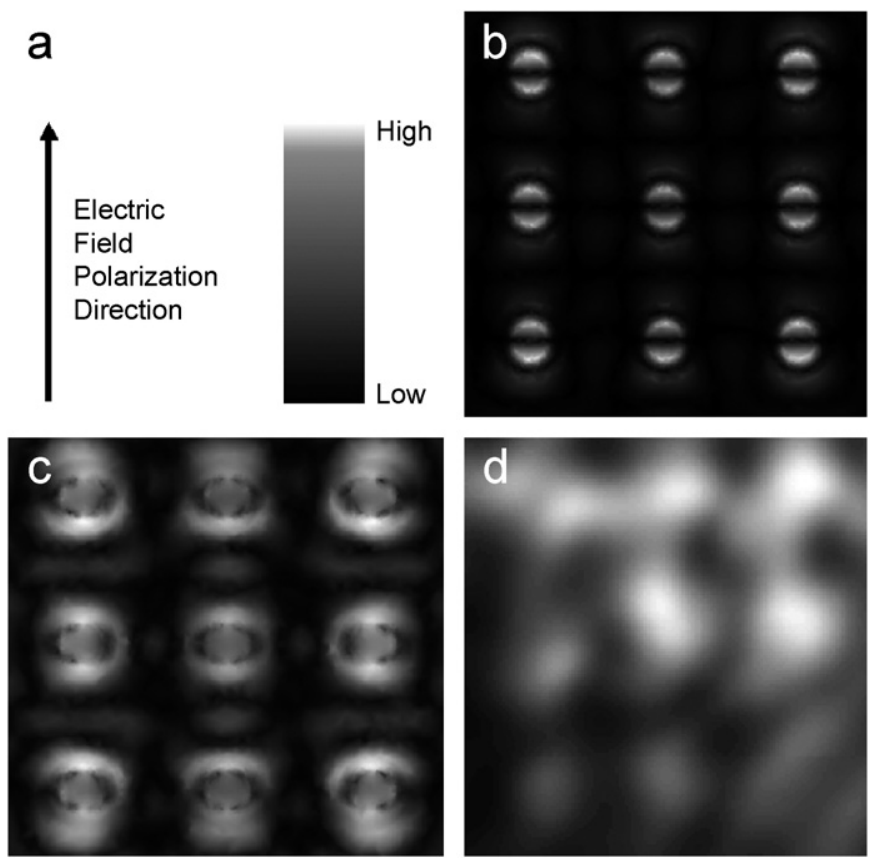

Fig. 5. (a) Incident electric field polarization and colorbar; (b) calculated $|E|_{\text {norm }} 1 \mathrm{~nm}$ above the surface; (c) calculated $|H|_{\text {trans }} 1 \mathrm{~nm}$ above the surface; (d) experimental SNOM data. Image size is $3 \times 3 \mu \mathrm{m}$.

$488 \mathrm{~nm}$ ) Nanonics tip with a $200 \mathrm{~nm} \mathrm{Cr}-\mathrm{Au}$ coating. Results from this experiment are shown in Fig. 5(d).

There are a number of factors contributing to the obvious differences between the calculated and measured near-field distributions shown in Fig. 5. Firstly, the interaction between the probe itself and the surface leads to significant distortions of the electromagnetic from that computed ignoring the probe. This distortion leads to much more complicated field patterns, which further complicates the interpretation of SNOM data. Secondly, the experimental data was recorded with the sample being illuminated with uncollimated light with an unknown polarization orientation. However, results from the FDTD calculation suggest that the polarization direction of the incident beam is responsible for the observed asymmetry. Hence, experimental results are expected to contain unknown asymmetries. Finally, it is standard practice in SNOM systems to oscillate the probe in a direction normal to the sample surface, in order to minimize damage to both the probe and the sample. However, the measured optical signal is an average over the full amplitude of the oscillation, which is of the order of $400 \mathrm{~nm}$. As shown in Fig. 6, the predicted field pattern depends strongly on the distance from the sample surface. Thus, the measured field pattern is a weighted average of the field pattern at all heights over the full amplitude of the oscillation. Work towards addressing this problem experimentally is ongoing.

\section{Conclusion}

In summary, a numerical model illustrating the high transmission efficiencies of silver composite metamaterials 

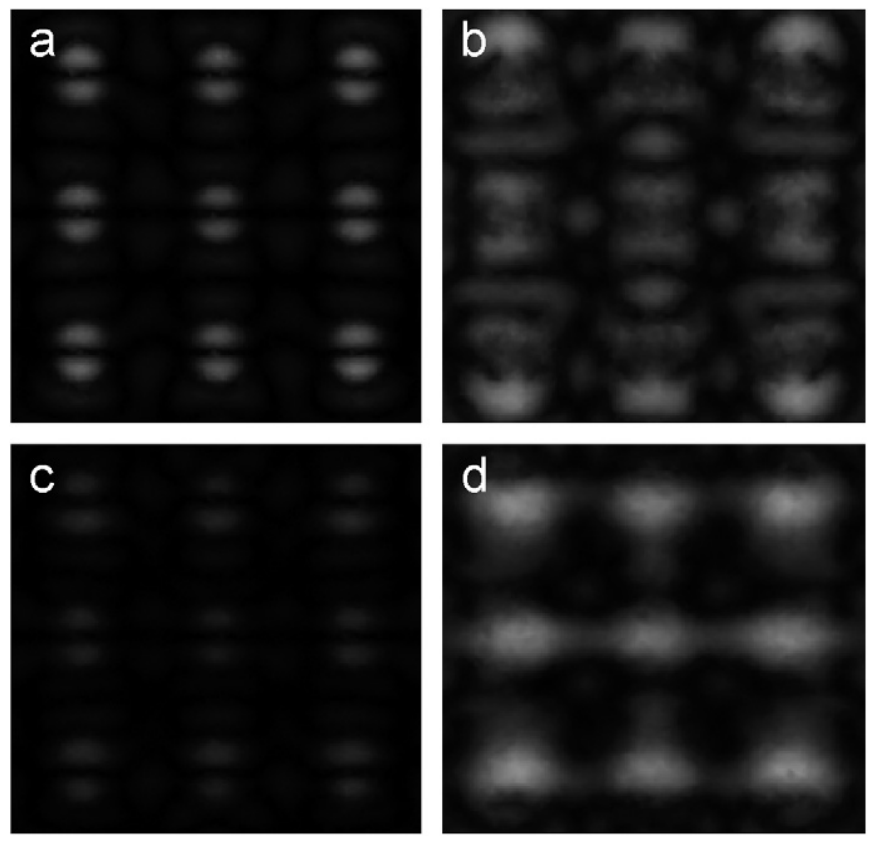

Fig. 6. Calculated electromagnetic field patterns: (a) $|E|_{\text {norm }} 100 \mathrm{~nm}$ above the surface; (b) $|H|_{\text {trans }} 100 \mathrm{~nm}$ above the surface; (c) $|E|_{\text {norm }} 200 \mathrm{~nm}$ above the surface; (d) $|H|_{\text {trans }} 200 \mathrm{~nm}$ above the surface. Image size is $3 \times 3 \mu \mathrm{m}$.

in the near infra-red regime is presented. Predictions from the model match experimental measurements in a semiquantitative manner for devices fabricated by FIB milling thin silver films.

FDTD modelling of the near-field behaviour of these materials shows highly localized electromagnetic fields, which qualifies these structures for potential applications for biosensing and platforms for nonlinear optics. Results from these simulations highlight the relevance of the probe sensitivity to various electromagnetic components, as a function of height above the surface. Progress towards experimental verification of the calculated near-field distributions is ongoing, with particular emphasis on the height dependence and field distortion caused by the SNOM probe.

\section{Acknowledgements}

The support of the Australian Research Council through its Centres of Excellence, Federation Fellow and Discovery programs is gratefully acknowledged. We also thank the Australian National University's Electron Microscopy Unit for use of the FIB.

\section{References}

[1] J.B. Pendry, Phys. Rev. Lett. 85 (2000) 3966.

[2] G. Raschke, S. Kowarik, T. Franzl, C. Sonnichsen, T. Klar, J. Feldmann, A. Nichtl, K. Kurzinger, Nano. Lett. 3 (2003) 935.

[3] E. Altewischer, M. van Exter, J. Woerdman, Nature 418 (2002) 304.

[4] Y. Yang, M. Nugami, J. Shi, H. Chen, G. Ma, S. Tang, App. Phys. Lett. 88 (2006) 81110.

[5] A. Roberts, R. McPhedran, IEEE Trans. Antennas Propag. 36 (1988) 607.

[6] F. Baida, D. van Labeke, Phys. Rev. B. 67 (2003) 155314.

[7] W. Fan, S. Zhang, B. Minhas, K. Malloy, S. Brueck, Phys. Rev. Lett. 94 (2005) 33902.

[8] F. Baida, D. van Labeke, G. Granet, A. Moreau, A. Belkir, Appl. Phys. B. 79 (2004) 1.

[9] A. Moreau, G. Granet, F. Baida, D. van Labeke, Opt. Exp. 11 (2003) 1131.

[10] D. Freeman, B. Luther-Davies, S. Madden, S. Stowe, Presented at 19th Aust. Conf. Microscopy \& Microanalysis, Sydney, February, 2006.

[11] Y. Poujet, M. Roussey, J. Salvi, F. Baida, D. van Labeke, A. Perentes, C. Santschi, P. Hoffmann, Phot. Nanostruct. Fund. Appl. 4 (2006) 47.

[12] E. Betzig, R. Chichester, Science 262 (1993) 1422.

[13] D. vanLabeke, D. Barchiesi, J. Opt. Soc. Am. A 10 (1993) 2193. 